\title{
Using Haptics to Convey Cause-and-Effect Relations in Climate Visualization
}

\author{
Nesra Yannier, Cagatay Basdogan, Serdar Tasiran, and Omer Lutfi Sen
}

\begin{abstract}
We investigate the potential role of haptics in augmenting the visualization of climate data. In existing approaches to climate visualization, dimensions of climate data such as temperature, humidity, wind, precipitation, and cloud water are typically represented using different visual markers and dimensions such as color, size, intensity, and orientation. Since the numbers of dimensions in climate data are large and climate data need to be represented in connection with the topography, purely visual representations typically overwhelm users. Rather than overloading the visual channel, we investigate an alternative approach in which some of the climate information is displayed through the haptic channel in order to alleviate the perceptual and cognitive load of the user. In this approach, haptic feedback is further used to provide guidance while exploring climate data in order to enable natural and intuitive learning of cause-and-effect relationships between climate variables. As the user explores climate data interactively under the guidance of wind forces displayed by a haptic device, she/he can understand better the occurrence of events such as cloud and rain formation and the effect of climate variables on these events. We designed a set of experiments to demonstrate the effectiveness of this multimodal approach. Our experiments with 33 human subjects show that haptic feedback significantly improves the understanding of climate data and the cause-and-effect relations between climate variables, as well as the interpretation of the variations in climate due to changes in terrain.
\end{abstract}

Index Terms-Data and information visualization, climate simulation, haptic exploration, multimodal user interfaces, human factors, haptic I/O, virtual reality, operator interfaces, commercial robots, and applications.

\section{INTRODUCTION}

$I^{\mathrm{N}}$ $\mathrm{N}$ this study, we investigate the integration of haptics and information visualization techniques in the exploration of complex multidimensional data. Our focus is the discovery and mastery of cause-and-effect relationships between patterns in data. The interactive exploration of climate data is our application domain. The large number of dimensions and constraints particular to this domain make it hard to devise a compelling purely visual representation that allows for an intuitive display of information and efficient exploration.

Traditional techniques in the visualization of atmospheric or climatic data make the processing of information difficult for scientists and for nonexperts who wish to learn about climate using real data. Data produced by climate simulation programs typically consists of values for a set of some 10 climate variables of primary interest such as temperature, humidity, wind intensity and direction, precipitation, and cloud water for a set of grid points in the atmosphere. Patterns in and relationships among these variables need to be examined in order to understand climate phenomena. Because climate data includes a large number of dimensions, different parameters are typically

- N. Yannier, C. Basdogan, and S. Tasiran are with the College of Engineering, Koc University, Sariyer, Istanbul 34450, Turkey.

E-mail:nesnes@stanford.edu, \{cbasdogan, stasiran\}@ku.edu.tr.

- O. Lutfi Sen is with the Eurasia Institute of Earth Sciences, Istanbul Technical University, Istanbul 34469, Turkey. E-mail: senomer@itu.edu.tr.

Manuscript received 6 May 2008; revised 7 Aug. 2008; accepted 30 Sept. 2008; published online 16 Oct. 2008.

Recommended for acceptance by $A$. Kheddar.

For information on obtaining reprints of this article, please send e-mail to: toh@computer.org, and reference IEEECS Log Number TH-2008-05-0031.

Digital Object Identifier no. 10.1109/ToH.2008.16. shown on different maps, requiring the integration of information from multiple maps for the interpretation of data. This may be especially difficult for nonexperts (i.e., students).

In meteorology, atmospheric parameters are usually depicted on hypothetical surfaces where pressure is constant at every point. A constant pressure surface is generally illustrated using contours in terms of its height from the mean sea level. These surfaces are not flat, but include ridges and troughs. Contour plots inherently make the perception of these features difficult.

Fig. $1 \mathrm{a}^{1}$ shows a relatively simple meteorological map, an 850-hPa geopotential height map (contours), for a particular day, obtained from a regional climate model simulation for the region surrounding Turkey. The plot also includes a depiction of the topography used in the model (color shade). An experienced meteorologist is expected to know how the wind blows on this surface and may guess where clouds form and, possibly, where this system generates precipitation. For those unfamiliar with such maps, it may be difficult to grasp and interpret even the basic aspects of the surface represented by these contours, let alone speculate about the wind characteristics and their effects. Fig. $1 \mathrm{~b}$ shows $850-\mathrm{hPa}$ wind fields (arrows) and precipitation (color shade) added to the plot given in Fig. 1a. In this figure, topography is excluded to avoid further complication. It would be useful for this plot to include more atmospheric parameters and surface features such as specific humidity, air temperature, cloud water,

1. The online version of the paper, which can be found on the Computer Society Digital Library at http://doi.ieeecomputersociety.org/10.1109/ ToH.2008.16, contains color copies of all figures. Details are more visible in the color version. 


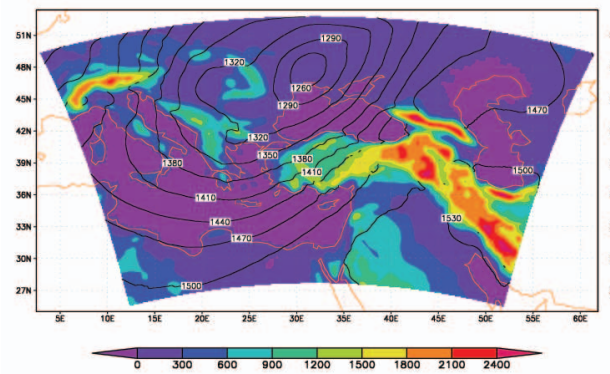

(a)

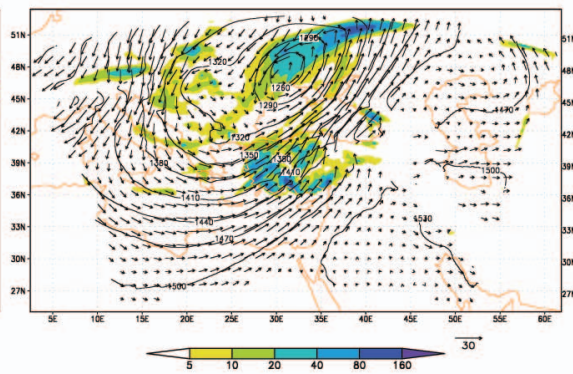

(b)

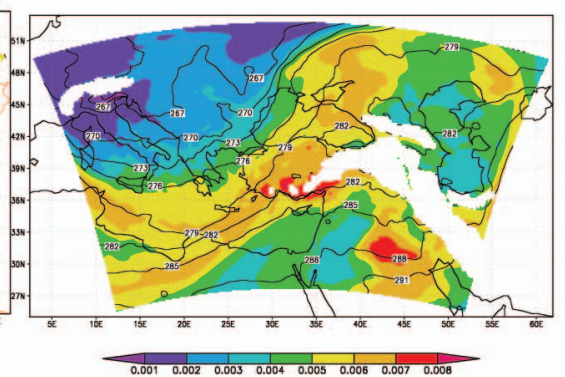

(c)

Fig. 1. Simulated weather conditions for a region covering southeastern Europe and part of the Middle East on a particular day created by GrADS. (a) Topography (in meters; shade) and $850-\mathrm{hPa}$ geopotential height (in geopotential meters; contours). (b) 850-hPa geopotential height (in geopotential meters; contours), 850-hPa winds (in meters per second; arrows) and precipitation (in millimeters per day; shade). (c) Specific moisture (in grams per kilogram; shade) and air temperature (in Kelvin; contours).

vorticity, and topography; however, in order to retain clarity, it is not desirable to include more than two or three parameters on the same plot. Instead, several separate plots for different parameters are generated. For example, Fig. 1c illustrates specific humidity (shade) and air temperature (contour) at $850-\mathrm{hPa}$ level for the same day. When all of the plots in Fig. 1 are analyzed together, they will provide an experienced observer with insight into the atmospheric conditions of this region on that specific day. However, the overwhelming visual information in these graphs makes it difficult for a nonexpert to integrate and interpret the data and come to a correct understanding of the meteorological conditions in the region on that day.

In addition to traditional visualization tools such as the Grid Analysis and Display System (GrADS) [1] and the tools developed by the National Center for Atmospheric Research (NCAR) [2], which generate data such as those presented above, there are also interactive forecast visualization tools such as IGrADS [3] used by the US Armed Forces or the Climate Visualization System (CLIMVIS) [4] used by NASA. Although these systems display information more effectively, they do not attempt to show all relevant data on one map, which would make it easier for users to identify and understand connections between the data without being overwhelmed.

Attempts have also been made to provide a more intuitive analysis of meteorological data through 3D Visualization Systems. Gateau [11], which allows atmospheric scientists to view $3 \mathrm{D}$ grid data without 3D programming knowledge, is one such tool. Its straightforward interface allows the user to interactively view atmospheric data in 3D [11]. Another method uses brush strokes to represent multidimensional data elements. In this method, the attributes of different data elements like precipitation, temperature, wind speed, and pressure are used to vary the appearance of the brush stroke's color, size, and orientation or texture [5]. Although these tools are successful at displaying more multidimensional data on the same graph, they do not adequately support user exploration or the discovery and understanding of the patterns in the data.

Multimodality may serve to communicate data to users more effectively by reducing the cognitive load placed on any one sense. Lawrence et al. have suggested that rendering modes that combine haptic and visual interfaces allow users to interactively explore scalar, vector, and tensor fields and facilitate their understanding of the data [12]. Harding et al. [6] have proposed combining 3D graphics, sonification and haptics into multisensory systems in order to manipulate geoscientific data interactively, which they suggest would facilitate many complicated tasks faced by geoscientists in the academia and industry. Jeong and Gluck have studied the feasibility of adding haptic and auditory displays to traditional visual geographic information systems [7].

While these multimodal systems serve the needs of scientists, Gordin and Pea have suggested that scientific visualization also has the potential to make science education more accessible and provide a means for scientific inquiry [13]. Edelson and Gordin [14] explain that scientific visualization technologies allow students to visualize scientific concepts using real complex data and to apply their knowledge in a discovery environment. They have applied this framework to the design of ClimateWatcher, a scientific visualization environment currently used in middle school, high school, and university settings [14]. ClimateWatcher displays surface temperature at a given time using color mapping. Although this software displays surface temperature effectively, it is not multimodal and does not show multiple levels of climate data at the same time. It also does not attempt to explain the cause-and-effect relationships among different climate data.

Although multimodal systems have been used to represent multidimensional data more intuitively, no attempt has been made to use a multimodal climate visualization system for education. Lee et al. have suggested that haptics can be used to display meteorological information [19], and Omata et al. have represented wind in climate data using vibrations and forces [18]. Our work also utilizes a multimodal approach but is distinct in that our goal is to convey causeeffect relationships in the domain of climate visualization and to enhance intuitive learning in this domain. The advantages of a multimodal approach in learning have been demonstrated in other fields. Haptic interfaces have been used to augment the teaching of physics, especially simple machines in elementary school [15], and the teaching of basic chemistry and structural biology has also been aided by haptic feedback enabling the user to touch different sizes and complexities of known molecular structures [16]. Feygin et al. have shown that haptic guidance helps the users 
remember a given task better by giving them a kinesthetic understanding [17]. Keeping in mind the role of haptics in education and in reducing the cognitive load in multidimensional data, we suggest that a multimodal climate visualization system can be constructed to help users understand the different dimensions of data and the causeand-effect relationships among them by taking advantage of the natural guidance provided by haptic feedback.

We present Climate Exploration and VIsualiZation (CEVIZ), a system for the presentation and interactive exploration of climate data [20]. CEVIZ is a prototype tool built to demonstrate how visual and haptic representations and haptic guidance can be used to improve upon purely visual representations. Design choices in CEVIZ were made in order to test the hypotheses given below. These design choices are based on the earlier findings that action and perception are coupled in haptics [21], [22] and humans can successfully memorize and cognize haptic stimuli [23], [25]. In addition, the research conducted by Ernst and Banks [26] shows that the human brain combines visual and haptic information in an optimal manner. While there was no strict rule followed in selecting the representations, generally, visual representations were determined to be more appropriate for the presentation of overview information while haptic representations were more useful in the representation of local phenomena. The hypotheses are stated as follows:

1. Haptics can be used to guide exploration along certain paths that correspond to cause-and-effect relationships and precedence relationships in time and, in this way, improve the discovery, learning, and retention of these relationships.

2. Haptic representations of certain kinds of data can reduce visual overload. Furthermore, augmenting visual representations with haptics improves the perception of data dimensions that do not stand out in purely visual representations.

3. Haptics can be used to help guide and/or confine exploration to areas where interesting phenomena occur. This improves the perception and recall of relationships between these phenomena.

4. Haptic representations provide significant improvement upon purely visual representations by enabling users to notice and remember relationships between different kinds of data variables when they are not paying explicit attention.

In addition to displaying the climate data to the user through visual and haptic channels, our work makes use of a number of information visualization techniques, for instance, the transparent information cube [8]. We discuss these techniques in Section 3 as we present how each climate data dimension is represented visually and/or using haptics. We do not introduce any novel visual representations; instead, we focus on picking a suitable combination of visual and haptic representations optimized to illustrate cause-and-effect relationships and minimize perceptual and cognitive overload.

We designed a set of human experiments in order to test the hypotheses listed above. In these experiments, the group that received visual and haptic feedback together consistently performed better at tasks than the other two groups that received visual feedback only. This and other results support our hypotheses.

\section{Preliminaries}

\subsection{Climate Data, Cause-and-Effect Relationships}

Climate data are provided by simulation programs at points that lie on pressure surfaces in the atmosphere. Conventionally, pressure surfaces at $500 \mathrm{mb}, 700 \mathrm{mb}, 850 \mathrm{mb}$, and $925 \mathrm{mb}$ are used to study climate data. These pressure surfaces may intersect with the terrain.

The output of a climate simulation program is a set of data points at a certain point in time (most often, a given day) on a particular pressure surface. Each data point consists of the following dimensions:

- $\quad x$ and $y$ coordinates (in degrees). These dimensions refer to the latitude and longitude of the point (shown from now on by $(x, y)$ ).

- p-level height (geopotential meter (gpm), the z coordinate, in kilometers). This dimension refers to the height (altitude) of the pressure surface at $(x, y)$.

- Precipitation (in millimeters). This dimension refers to rain, snow, and other forms of water falling at $(x, y)$.

- Air temperature (in Kelvin). This dimension refers to the measure of the heat content of the air at $(x, y, z)$.

- Zonal wind (in meters per second). This dimension refers to the component of the wind velocity at $(x, y, z)$ along the local latitude, i.e., the $x$ component.

- Meridional wind (in meters per second). This dimension refers to the component of the wind velocity at $(x, y, z)$ along the local longitude.

- Specific moisture (in kilograms per kilogram). This dimension refers to the humidity of the air at $(x, y, z)$.

- Cloud water (in kilograms per kilogram). This dimension refers to the total concentration of ice and liquid water particles in a cloud, if one exists, at $(\mathrm{x}, \mathrm{y}, \mathrm{z})$.

- Vorticity (in Kelvin square meters per kilogram second). This dimension refers to the force defined as the curl of the wind velocity representing the amount of circulation or rotation. Vorticity is a force perpendicular to the plane along which the wind velocity is given. The vorticity makes the wind rise or fall due to the circulation of the air.

The following is a list of noteworthy interactions and cause-and-effect relationships among climate data variables and the terrain:

Clouds form when wind has enough humidity and enters into a relatively cooler region.

Clouds occur in regions where winds collide with mountains.

- Clouds occur in areas of humidity where there is counterclockwise wind circulation in the northern hemisphere and where there is clockwise wind circulation in the southern hemisphere.

- For precipitation to occur, humid air must rise. Furthermore, there must be a significant temperature drop from a higher pressure level (closer to the earth's surface) to a lower pressure level. 
Learning and internalizing these basic climate-related principles becomes important in two settings. First, while climate nonexperts are being taught climate phenomena, they need to develop a working knowledge of these principles in order to be able to use them without much cognitive cost. This is especially the case when they are interpreting climate patterns and climate data at different points in time or for different geographical configurations. Second, while climate experts are analyzing and comparing results of climate simulations, it is desirable to convey to them particular occurrences of the above relationships in a way that does not demand too much of their conscious cognitive attention. This allows them to instead focus on what is different about a particular set of climate data. Demonstrating that haptics-augmented visualization techniques facilitate this learning and internalization is the primary contribution of our work.

\subsection{Climate Simulation}

For the experiments we report in this paper, we use climate data provided by the simulation program RegCM3 [9]. RegCM3 makes use of a three-dimensional sigma-coordinate primitive equation climate model.

The simulation program RegCM3 takes boundary and initial conditions as input, which are the specific data values in the region of interest corresponding to the day the climate simulation is started. These include the description of the terrain in the form of a height map, i.e., a set of points of the form $(x, y, z)$, where $z$ is the altitude of the terrain (in kilometers) above sea level at coordinate $(x, y)$, and $x$ and $y$ are given as grid points along west-east and north-south directions, respectively. The boundary and initial conditions, including the height map and the vegetation cover for the region we investigated, were provided by the Eurasia Earth Sciences Institute (AYBE), Istanbul Technical University. RegCM3 can simulate the evolution of the data variables for various lengths of time.

In order to investigate the effect of vegetation or certain terrain features, climatologists run simulations of the same geographic area with different vegetation or at different points in time or with particular terrain features modified. The results of such sets of simulations are data files of the same format. In such cases, climatologists are interested in comparing macropatterns in climate data and finding differences in climate events.

\section{The CEVIZ System}

The CEVIZ system takes as input the climate data files produced by RegCM3 and, by using a combination of haptic and information visualization techniques, presents them in an interactive way to the user (Fig. 2). Details of how each kind of climate data is presented are provided in Section 3.1. The design choices in the CEVIZ system were arrived at after some experimentation with earlier prototypes. The choice of where and how to use haptics and where to use point rather than overview information, as well as the particulars of how each dimension was represented, was made so that a comparison between a purely visual system and a haptics-augmented system would allow us to make conclusions about hypotheses 1-4 explained in the Introduction.

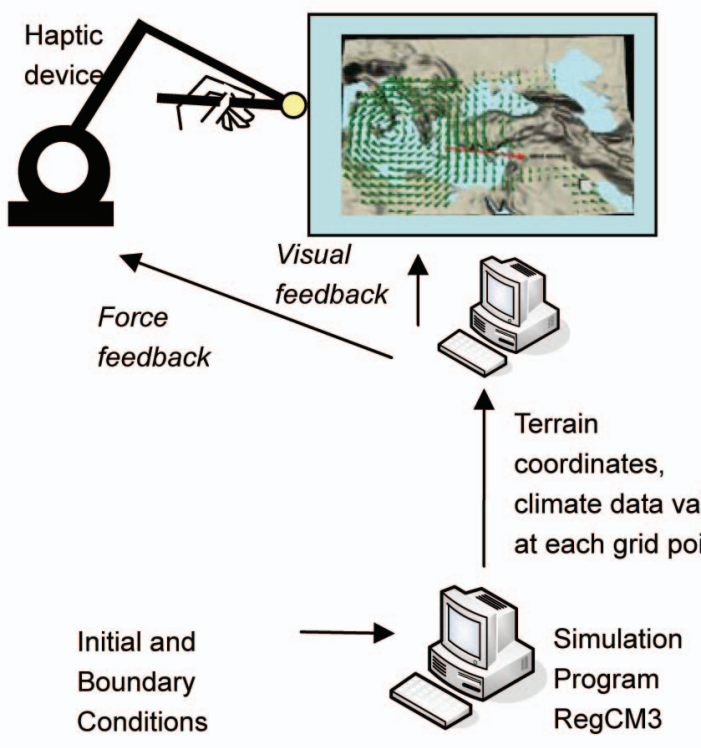

Fig. 2. Block diagram of the CEVIZ system.

\subsection{Representation of Climate Data}

For each kind of climate data listed below, we explored several visual, haptic, and combined representation alternatives. Due to space limitations, we discuss here only a few of the alternatives that were considered and discarded. In all cases, the mapping from climate variables to visual and haptic quantities was linear.

Height map. A three-dimensional mesh representation of topography is formed by connecting the height values for each grid point using the height map provided by AYBE. Height values are mapped to color shades ranging from blue (sea level) to gray. The height map is also used for haptic rendering.

Pressure surface. The p-level height data are used to construct a pressure surface. This surface is not shown visually because it blocks the user's view of the terrain too much. Instead, it is mapped as an invisible haptic surface that the user can feel and move along (see the review of haptic rendering concepts in [24]). Only the parts of the terrain above the pressure surface being explored can be felt by the user.

Wind. A combination of visual and haptic representations is used for winds. The visual representation consists of conical arrows at grid points (Fig. 3). The apex of the cone points in the direction of the wind, and the radius and height of the cone are both proportional to the magnitude of the wind. At the beginning of exploration, none of the wind arrows are displayed. The wind arrows at grid points within a certain distance of the cursor are made visible as the terrain is explored.

Forces rendered through the haptic device lead the cursor and guide the user to follow the path of the winds. This is implemented by dividing the map into gridlines on the xy plane and assigning a force vector to each point in the grid. The zonal and the meridional winds are mapped as forces in the $\mathrm{x}$ and $\mathrm{y}$ directions. Forces at points intermediate between grid points are computed by a linear interpolation of the forces at the neighboring four vertices on the grid. We initially experimented with displaying all 


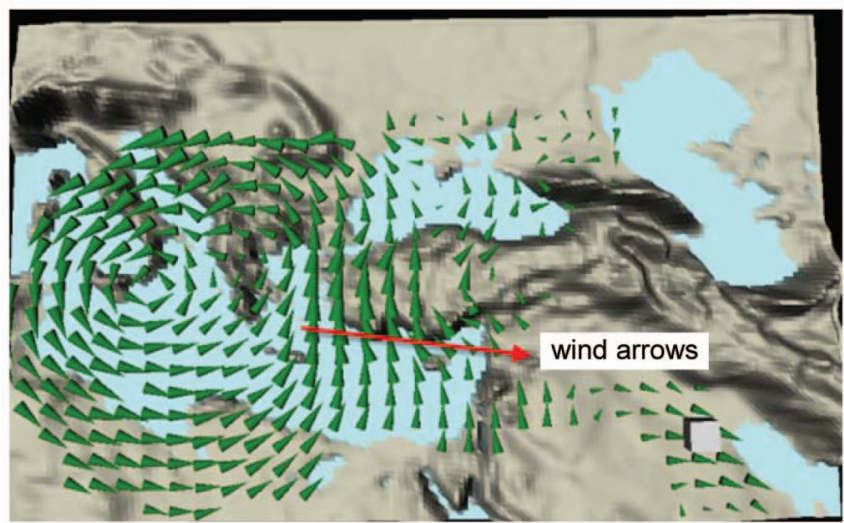

Fig. 3. Representation of the winds in the CEVIZ system.

of the wind data at once statically throughout the entire map and found that rather than allowing the user to grasp wind overview information rapidly, this representation instead overloads the user and makes it more difficult to retain this information. We found that when the entire overview is presented at once, the user typically is unable to immediately absorb the overview and instead serially explores the map.

We tested if displaying wind information gradually, while the user is guided by the wind forces displayed through a haptic device, gives the impression of exploring other climate variables while traveling with the wind. As our experiments demonstrate, this was instrumental in communicating cause-effect relationships because, as explained in Section 2.1, the effect is always located downstream in the wind and happens after the phenomenon that causes it.

Cloud water. Cloud water is represented graphically by white spheres mapped over the pressure surface with radii directly proportional to the cloud water value at that point (Fig. 4). In a quick overview of the terrain, the regions where the spheres are largest and most frequent easily stand out as the regions where there is the largest amount of cloud water.

Cloud water is also represented haptically using bump textures mapped onto the pressure surface [10]. The size of the bumps is proportional to their radii.

Humidity and air temperature. The cursor in CEVIZ is an information box. Humidity (specific moisture) is represented

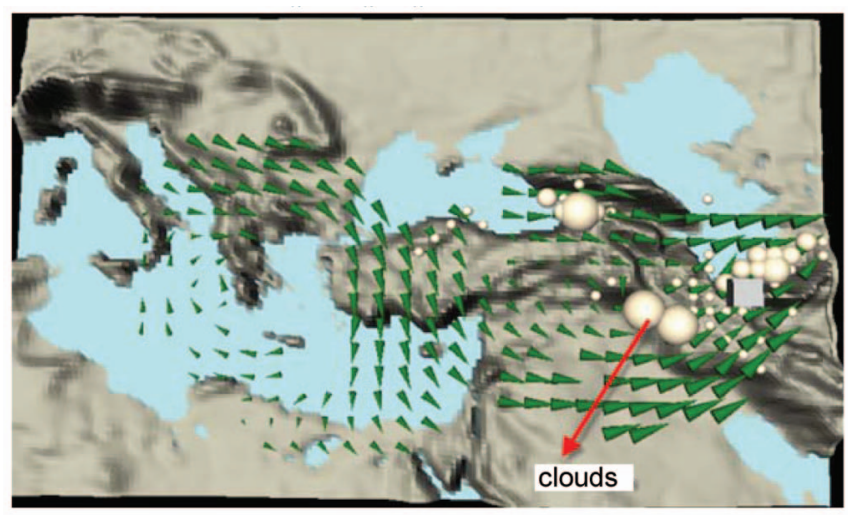

Fig. 4. Cloud water represented by the sizes of white spheres.

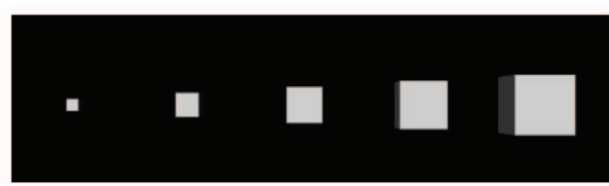

less humid

more humid

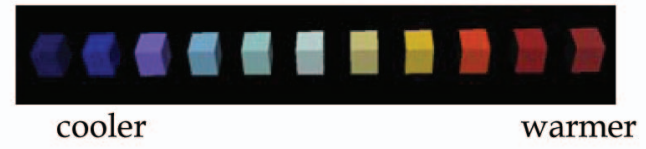

Fig. 5. Humidity and temperature are mapped to the size and color of the information box.

by the size of the information box. There is a linear mapping from the moisture values to the size of the box. Temperature values are represented by the color of the information box. Air temperature is divided into 11 intervals, and each interval is assigned a color (Fig. 5).

The information cube lets the user observe these variables at specific points rather than giving an overview for the whole map. This draws the user's attention to changes in climate data, while the cursor motion is guided by the haptic forces. The user experiences these changes in humidity and temperature that are caused by or happen concurrently with the wind motion. This design choice was made in order to convey cause-and-effect relationships more explicitly. The information cube has a shadow (Fig. 6) directly beneath it on the terrain, which is used to show the exact location of the information box over the xy plane.

Rain. Rain is illustrated by animated small black (blue) cones with radii directly proportional with the precipitation value (in millimeters) at that point (Fig. 7).

Vorticity and rain. When there is a counterclockwise circulation in the northern hemisphere or when the wind collides with the mountains, air tends to rise. In our system, vorticity is represented by a resistive spring force $\mathrm{F}=-\mathrm{k} * \Delta \mathrm{z}$ at each point, with the magnitude of the spring constant $\mathrm{k}$ being inversely proportional to the vorticity force and $\Delta \mathrm{z}$ representing the distance the cursor lifts up from the pressure surface. As a result, it is easier for the user to lift the cursor from a pressure surface in regions where vorticity is high. We chose to render vorticity only in

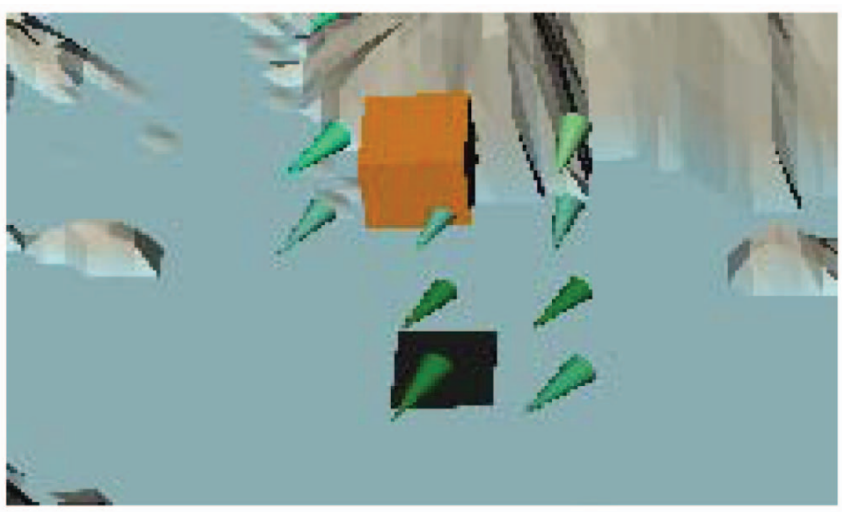

Fig. 6. The shadow of the information cube shows the location of the cursor on the terrain. 

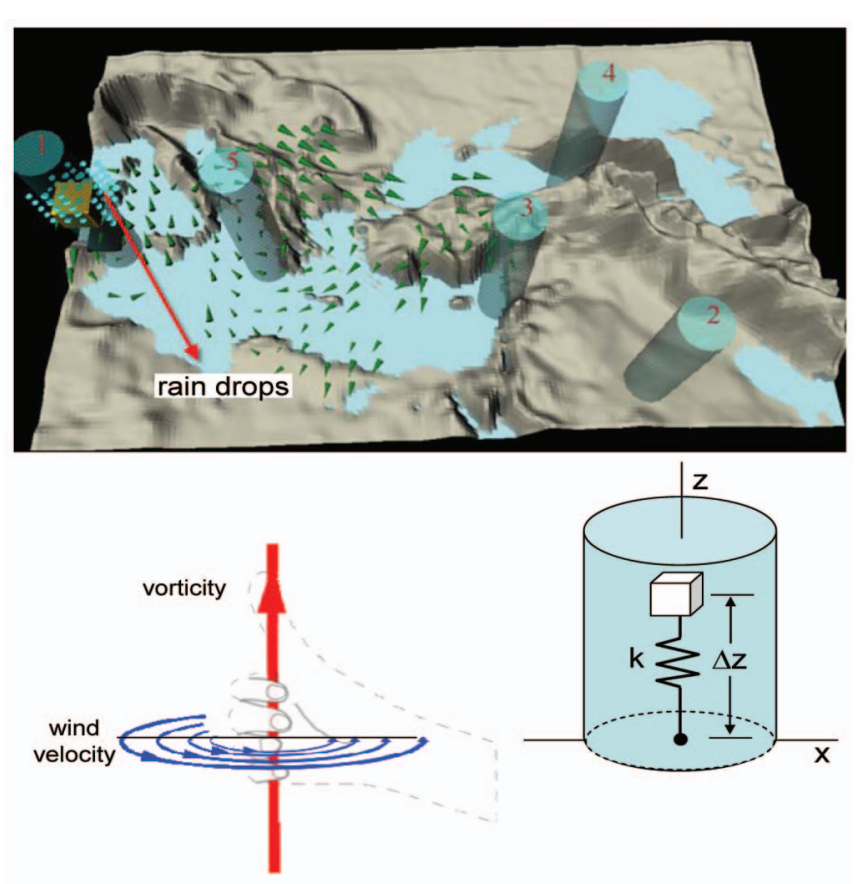

Fig. 7. The representation of rain cylinders and raindrops. The cylinders represent the areas where vorticity and precipitation are high. Within the cylinders, a spring force inversely proportional to vorticity pulls the cursor toward the pressure surface.

the areas where vorticity and precipitation are high. In these areas, vorticity causes air to rise. These areas were delineated by black (blue) cylinders (Fig. 7).

When the cursor is outside but close to the boundary of one of the cylinders, haptic forces pull the cursor into the cylinder with a spring force proportional to the distance of the cursor from the cylinder surface. Once inside the cylinder, lateral spring forces help keep the cursor confined within the cylinder. As the user tries to lift up the cursor while inside the cylinder, he/she moves inside a tunnel (a virtual fixture) along a vertical line going up from that point.

When the cursor is inside a cylinder, if there is enough humidity, rain starts to fall. The temperature and drop in humidity as the cursor rises in the cylinder are represented by the changes in the color and size of the information cube. Since pressure is directly proportional to temperature by the ideal gas law, as the air rises, the pressure drops and causes the temperature to drop as well.

In the cylinders where rain forms, the user observes raindrops moving up and down as he or she moves the information cube up and down. When the cursor arrives at a pressure surface, the raindrops disappear, and when it is lifted, they reappear. This design choice was made to make more explicit the fact that rain forms when the wind rises.

We experimented with other ways of representing vorticity but found them to be counterintuitive. For instance, we tried rain tunnels that had a force pulling the cursor up along the axis of the cylinder and moving him or her to a pressure surface above the current one, at which point the climate data in the newly reached pressure surface would be displayed. We found that the pull felt very abrupt to users, and it was very difficult for them to notice that they had moved up to a pressure surface at a higher altitude. They would be confined to this new pressure surface and haptic forces would work against them if they wanted to repeat the transition from one pressure surface to the other. Users felt that this was counterintuitive.

\section{Experimental Evaluation and Discussion}

In order to test our hypotheses, we designed five experiments in which the user explores the climate system around Turkey for different time periods and attempts to figure out the cause-and-effect relationships between different geographic and climatic variables.

We used climate data files produced by RegCM3 for the region surrounding Turkey. Climate data variables were provided on a grid in the xy plane with $24 \mathrm{~km}$ in between each grid point. There were 169 grid points along the $\mathrm{x}$ direction and 115 grid points along the $\mathrm{y}$ direction.

Thirty-three human subjects participated in the experiments. Subjects were divided into three groups (11 in each group). Only visual feedback (V) was provided to the subjects in Groups 1 and 2, whereas both visual and haptic feedback $(\mathrm{V}+\mathrm{H})$ were provided to the subjects in Group 3.The subjects in Group 1 (represented by V1 in the text) held the stylus of the haptic device and freely explored the surface of the map with no force feedback. For subjects in Group 3, haptic feedback guided the exploration, and certain climate parameters and the terrain were rendered using haptics.

To subjects in Group 2 (appears as V2 in the text), instead of allowing them free and unguided visual exploration, the climate data were presented along the paths of cause-andeffect relationships in a sequence that reflected the occurrence and progress of these relationships in time. This was accomplished by showing them selected videos of subjects from Group 3. For this reason, in the experiments below, we do not provide a separate description for subjects in Group 2.

The purpose of the experiments performed by subjects in Group 2 was to test whether the improvement brought about by haptics is almost completely due to the fact that exploration is guided along particular paths or whether haptics provides observational and learning benefits beyond this.

\subsection{Experiment 1: Wind}

Goal. The goal of this experiment is to investigate whether haptics helps a user observe and remember wind patterns better.

Stimuli. In the visual version of this experiment (V1), as the user explores the surface, he or she sees the arrows appearing gradually on the screen. The user's goal is to explore the whole surface until all the arrows are visible on the screen (Fig. 8).

In the haptics-enhanced version $(\mathrm{V}+\mathrm{H})$, in addition to seeing the wind arrows, the user feels the wind forces in real time through the haptic device. Also, haptic feedback snaps the cursor onto the pressure surface during the exploration by applying a spring force when the user wants to move the cursor away from the pressure surface.

Procedure. The users are instructed to explore the whole surface until all the arrows are visible on the screen. At that point, the visual and haptic displays are turned off, and the users are given six different maps representing different wind patterns (see Fig. 9 for examples) and asked to choose and rank the three maps that most closely match the one they explored. 


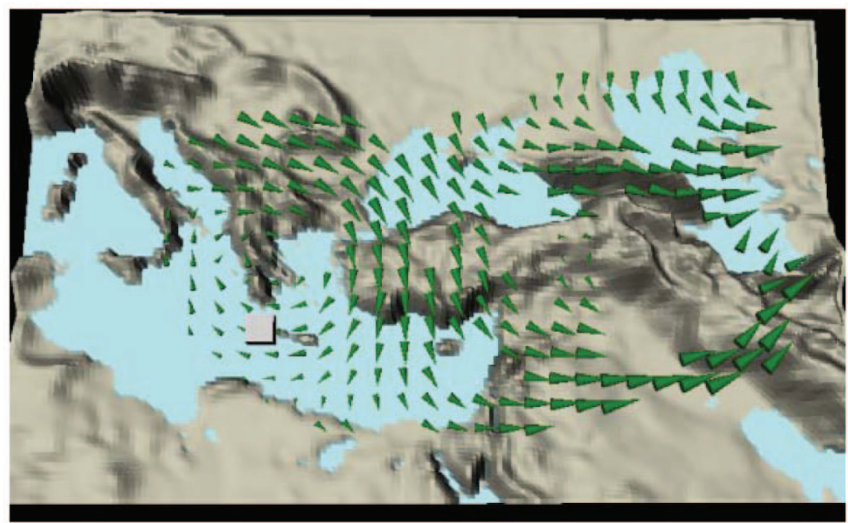

Fig. 8. The user exploring the patterns of wind in experiment 1.

Results. The columns of the table below titled first, second, and third represent the number of subjects in each group who chose the correct answer as their first, second, and third choice. The column titled raw score is the number of subjects that chose the right map in any one of their three choices. The penalty-based score is calculated by assigning three, two, and one points to the first, second, and third choices.

\begin{tabular}{|c|c|c|c|c|c|}
\hline & 1st & 2nd & 3rd & Raw Score & $\begin{array}{c}\text { Penalty-based } \\
\text { Score }\end{array}$ \\
\hline V1 & 5 & 2 & 0 & $7 / 11(64 \%)$ & $19 / 33(58 \%)$ \\
\hline V2 & 2 & 3 & 2 & $7 / 11(64 \%)$ & $14 / 33(42 \%)$ \\
\hline V+H & 6 & 2 & 0 & $8 / 11(73 \%)$ & $22 / 33(67 \%)$ \\
\hline
\end{tabular}

\subsection{Experiment 2: Cloud Formation}

Goal. The statement "When wind has enough humidity and enters a relatively cooler region, clouds form" specifies a cause-and-effect relationship between climate variables. The goal of this experiment was to test whether the combined haptic and visual approach improves upon a purely visual approach in learning this relationship and later being able to use it to make inferences.

Stimuli. In the visual version of this experiment (V1), as the user explores the surface, wind arrows start appearing. Five locations are marked by numbers and off-white spheres on the map (Fig. 10), and the user is asked to choose the regions where clouds are likely to occur. In the haptic version of the experiment, the same visual representations are used for temperature and humidity with the addition of the haptic feedback for representing the winds. The wind forces guide the user to follow the path of the winds and observe the changes in the climate variables along these paths.

Procedure. The subjects are given a handout explaining that they will investigate the cause-and-effect relationships
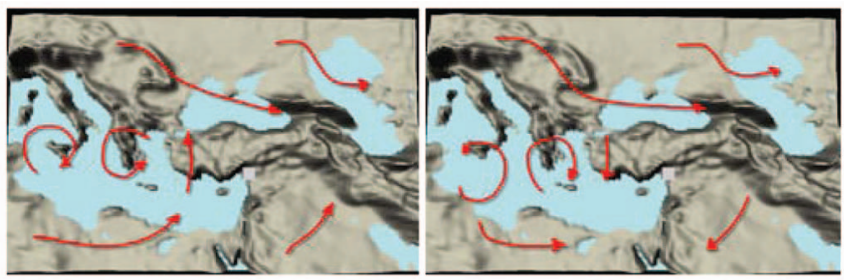

Fig. 9. Two of the six wind pattern map choices in experiment 1.

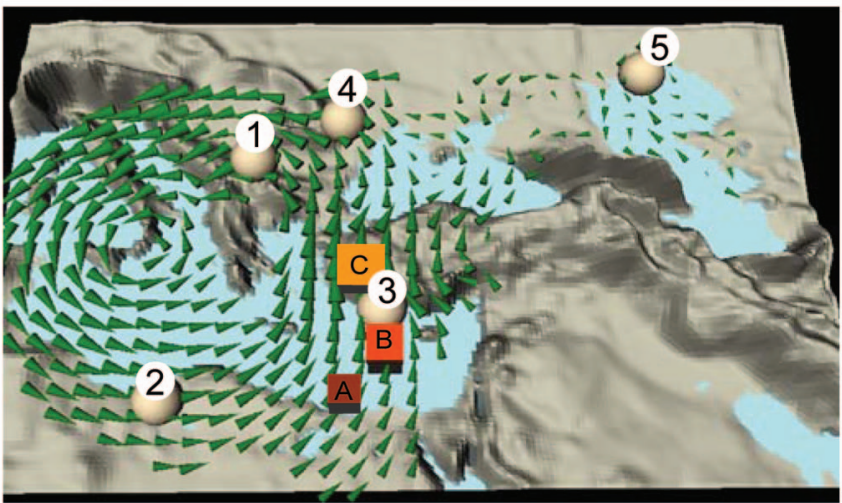

Fig. 10. Winds and clouds in experiment 2. The addition of haptics introduces a sense of direction for understanding causalities in the climate data. For example, if the user follows the path A-B-C (along the direction of winds), the color of the cube changes from dark to light, indicating a drop in temperature, and its size gets bigger, indicating an increase in humidity. Hence, clouds form in location number 3.

between humidity, wind, temperature, and formation of clouds in the experiment. They are given the statement "Wind carries humidity. If the wind has enough humidity and enters into a relatively cooler region, clouds form." The subjects are then asked to explore the pressure surface and decide in which of the five regions (provided to them in the beginning of the experiment) clouds are likely to form. Subjects are allowed to choose as many regions as they like, and winds are rendered throughout the experiment.

Results. The results of this experiment are shown in the table below. The perfect scores represent the number of participants that gave the perfect answer (1-3-4), while the raw scores are based on the number of correct answers only. The penalty-based scores are calculated by assigning " +1 " to each region chosen correctly and " -1 " to each incorrectly chosen.

\begin{tabular}{|c|c|c|c|}
\hline & Perfect Score & Raw Score & Penalty-based Score \\
\hline V1 & $2 / 11(18 \%)$ & $25 / 33(76 \%)$ & $21 / 33(64 \%)$ \\
\hline V2 & $3 / 11(27 \%)$ & $25 / 33(76 \%)$ & $20 / 33(61 \%)$ \\
\hline V+H & $5 / 11(45 \%)$ & $31 / 33(94 \%)$ & $24 / 33(73 \%)$ \\
\hline
\end{tabular}

\subsection{Experiment 3: Locating Clouds}

Goal. The goal of this experiment is to investigate whether haptic feedback helps the user construct a link between the cloud location, the wind patterns, and the terrain. Differently from experiment 2, no statement is given to the subjects about the relations between climate variables in order to test whether haptics helps users learn without explicitly paying attention.

Stimuli. All versions of this experiment (V1, V2, and $\mathrm{V}+\mathrm{H}$ ) consist of a learning phase followed by a testing phase.

In the learning phase of the visual version of the experiment (V1), the user observes clouds appearing on the surface together with the wind arrows. First, the user explores the whole surface until all the arrows and clouds are visible on the screen (Fig. 11). In the testing phase, the user is given the same system, but this time without the clouds appearing on the surface and is asked to guess the location of three cloud clusters in five trials each. During this second part of the experiment where the user's learning is being tested, as 


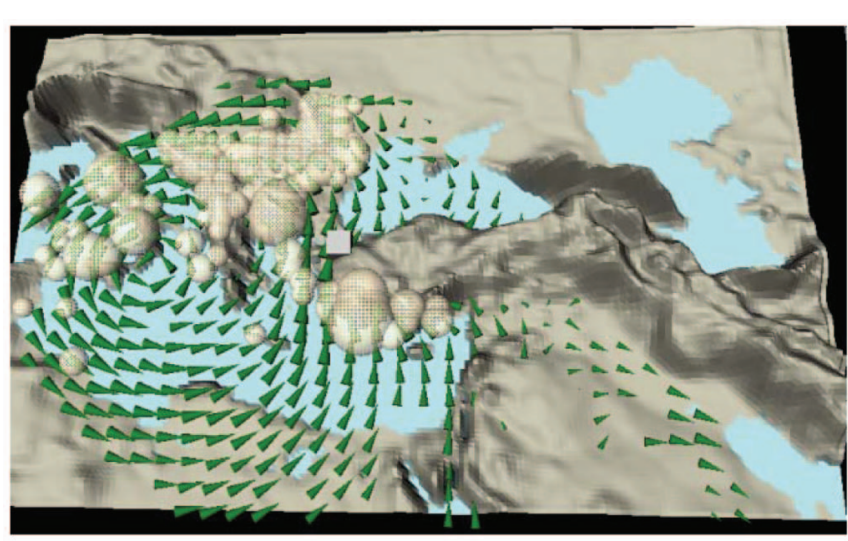

Fig. 11. Winds and clouds in experiment 3.

the user explores the surface, only the wind arrows appear on the screen gradually. The user is asked to find the location of the cloud clusters.

In the haptic version, haptic feedback enables the user to feel the winds, the pressure surface, and the terrain. Also, a bump texture is haptically mapped (as explained in Section 3) onto the surface to represent the clouds during the exploration along with the visual sphere representations. The testing phase of the haptic version is the same as that of the visual version. Haptic feedback is on throughout the experiment.

Procedure. Once all the arrows and clouds fully appear on the screen, the training phase of the experiment is over, and haptic and visual displays are turned off. The participant is then asked to pick which of the following statements is true:

a. Clouds occur in regions of clockwise circulation.

b. Clouds occur in regions of counterclockwise circulation.

c. Clouds don't occur in regions of circulation.

d. Clouds occur both in regions of clockwise and counterclockwise circulation.

e. Clouds occur in regions where the wind collides with the mountains.

Results. The correct answer for the first part of the experiment was the choices " $b$ " and "e." The distribution of the subjects' responses is given below. The penalty based score was calculated by assigning " +1 " to each correct answer (i.e., to each " $b$ " or " $e$ " chosen by the user) and " -1 " is assigned to each wrong answer (i.e., to each " $a$, , " $c$," or " $d$ " chosen by the user).

\begin{tabular}{|c|c|c|}
\hline & Raw Score & Penalty-based Score \\
\hline V1 & $4 / 11(36 \%)$ & $-3 / 22(-14 \%)$ \\
\hline V2 & $8 / 11(73 \%)$ & $10 / 22(45 \%)$ \\
\hline V+H & $10 / 11(91 \%)$ & $12 / 22(55 \%)$ \\
\hline
\end{tabular}

\subsection{Experiment 4: Rain}

Goal. The aim of this experiment is to investigate whether haptic feedback helps the user realize that the air must rise (vorticity being positive and high) in order for rain to occur and observe the changes in other climate variables, like temperature and humidity, during the rain formation.

Stimuli. Five cylinders in vertical position are displayed to the user on the screen (Fig. 7, Section 3). If the cursor enters a cylinder, the user can investigate whether or not rain forms in the cylinder. The five cylinders are located so that each has different characteristics in terms of humidity, vorticity, wind pattern, and rain formation.

In the haptic version of the experiment, as described in Section 3, when the cursor is close to a cylinder, haptic forces draw the cursor inside the cylinder. While inside the cylinder, as the user moves the cursor up, he or she feels side forces inhibiting him or her from going out of the cylinder creating a tunnel effect. In the cylinders where the vorticity is high and the forces allow him or her to lift the cursor, rain starts to fall if the air has enough humidity, i.e., if the information box is large. The user is also able to feel the circulating wind forces around the cylinder if the cursor steps outside the cylinder.

The visual version of the experiment (V1) is similar, except that the user is not able to feel any forces. In order to create a visual effect that matches the haptic interaction as closely as possible, we adjust the positional displacements of the visual cursor based on the vorticity values inside cylinders. When the vorticity force is weak, the visual cursor does not move up much in response to the movements of the user's hand. If vorticity is low, until a certain cursor displacement threshold is exceeded, the visual cursor does not move at all in response to the movement of the stylus.

Procedure. At the end of the exploration, the user is asked to answer the following questions for each cylinder:

$\mathrm{Q}$ 4.4.1. Why does/doesn't rain occur in Cylinder " $n$ "?

A. The temperature difference between the pressure surfaces is/isn't significant.

B. The humidity when the cylinder is entered is high/low.

C. The wind inside the cylinder is/is not able to rise.

$\mathrm{Q}$ 4.4.2. Which of the below are true for Cylinder " $n$ "?

a. There is clockwise wind circulation.

b. There is counterclockwise wind circulation.

c. There is no circulation.

d. The wind collides with the terrain.

e. The wind doesn't collide with the terrain.

The user is then asked to circle the correct answers in the following questionnaire deducing from the information gained in the experiment.

Q 4.4.3.

Rain occurs if

a. air can/cannot rise.

b. there is clockwise/counterclockwise circulation.

c. wind collides with terrain/There is no circulation and wind does not collide with the terrain.

d. there is/is not a significant temperature drop.

e. wind has/does not have significant humidity.

When rain occurs, humidity:
a. drops
b. rises
c. stays the same.

Then, a new set of climate data on the same terrain, this time with four different cylinders is displayed. The user is asked to pick in which of the cylinders rain is likely to form. 


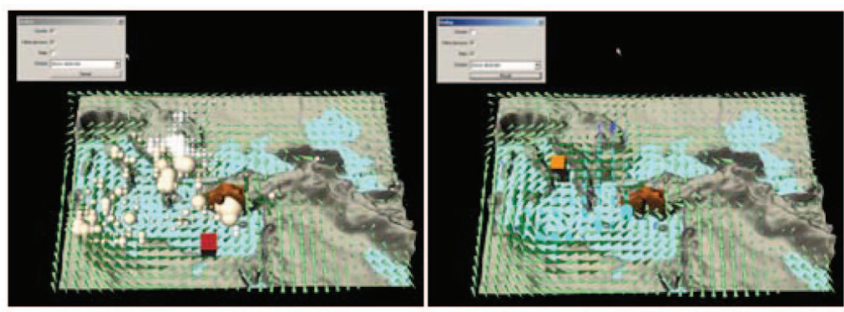

Fig. 12. Cloud and rain patterns in experiment 5.2. The heights of the mountains in the south coast of Turkey on the Mediterranean Sea (see the darker region on the map) are intentionally increased to change the climate conditions.

The user is also given the picture of the previously explored system (i.e., the training system) and is asked to respond to the following question for each cylinder in the new system:

Q 4.4.4. Cylinder "?": No/little/a lot of rain will occur.

$\mathrm{Q}$ 4.4.5. Which cylinder shown in the training system is similar to this cylinder? Give two answers you think match best.

Results. The percentage of correct answers for each question is given for both categories of subjects below. In questions Q4.4.1, Q4.4.2, Q4.4.4, and Q4.4.5, the standard deviations show the variations in subjects' response among cylinders. In Q4.4.3, the standard deviations show the variations in subjects' response to the six different questions given above. In Q4.4.5, the first number is the raw score, while the second one is the penalty-based score. The penalty-based score is calculated by assigning " +2 " to each correct answer chosen as the best match and " +1 " to each correct answer chosen as the second match.

\begin{tabular}{|c|c|c|c|c|c|}
\hline & $\mathrm{Q} 4.4 .1$ & $\mathrm{Q} 4.4 .2$ & $\mathrm{Q} 4.4 .3$ & $\mathrm{Q} 4.4 .4$ & $\mathrm{Q} 4.4 .5$ \\
\hline $\mathrm{V} 1$ & $6.53 / 11$ & $7.80 / 11$ & $8.17 / 11$ & $4.00 / 11$ & $(30 \pm 11 \%)$ \\
& $(59 \pm 14 \%)$ & $(71 \pm 25 \%)$ & $(74 \pm 11 \%)$ & $(36 \pm 26 \%)$ & $(32 \pm 12 \%)$ \\
\hline V2 & $7.87 / 11$ & $7.60 / 11$ & $9.50 / 11$ & $8.50 / 11$ & $(48 \pm 20 \%)$ \\
& $(72 \pm 9 \%)$ & $(69 \pm 19 \%)$ & $(86 \pm 21 \%)$ & $(77 \pm 10 \%)$ & $(35 \pm 15 \%)$ \\
\hline $\mathrm{V}+\mathrm{H}$ & $10.53 / 11$ & $8.20 / 11$ & $8.83 / 11$ & $8.50 / 11$ & $(43 \pm 11 \%)$ \\
& $(96 \pm 7 \%)$ & $(75 \pm 21 \%)$ & $(80 \pm 21 \%)$ & $(77 \pm 34 \%)$ & $(41 \pm 12 \%)$ \\
\hline
\end{tabular}

\subsection{Experiment 5: Terrain Manipulations}

Goal. The visual representations of climate data "before" and "after" terrain manipulation shown on the same map overwhelm the user. This experiment investigates if haptic feedback helps the user understand and interpret the differences between two maps.

Stimuli. In experiment 5.1, the mountains in the northeast region are removed. In experiment 5.2, the heights of the mountains in the Antalya region (south coast of Turkey on the Mediterranean Sea) are increased (see Fig. 12). The information appearing on the first window is data for the unmodified terrain. As the user explores the surface, the representation of the climate data corresponding to the modified terrain replaces the original data in areas within certain proximity of the cursor. The user can reset the map to display the original data at any time. He or she can also turn on/off the visual representations for clouds, rain, and wind. In the haptic version of the experiment, haptic feedback guides the user to explore climate changes along paths of strong winds. As usual, the user is able to feel the terrain surface in addition to the winds.

Procedure. The user is initially given the original system and asked to explore the surface to observe the changes. As the user explores the terrain, new climate data are rendered in the areas explored by the cursor. After the user is finished exploring, she/he is asked to explain the changes by answering the following question, based on the knowledge he or she has gathered from the earlier experiments:

What feature in the "after" version of the climate data do you think played a role in the changes that occurred? (You can choose more than one answer)

a. clockwise circulation of the wind

b. counterclockwise circulation of the wind

c. no circulation of the wind

d. wind collides with terrain.

Results. The percentage of correct answers by $\mathrm{V}$ and $\mathrm{V}+\mathrm{H}$ subjects in experiments 5.1 and 5.2 are shown in the table below.

\begin{tabular}{|c|c|c|}
\hline & Exp. 5.1 & Exp. 5.2 \\
\hline V1 & $7 / 11(64 \%)$ & $6 / 11(55 \%)$ \\
\hline V2 & $9 / 11(82 \%)$ & $7 / 11(64 \%)$ \\
\hline V+H & $9 / 11(82 \%)$ & $11 / 11(100 \%)$ \\
\hline
\end{tabular}

\section{Discussion}

The results of experiment 1 show that haptic feedback improves user recall of wind direction. The task was very challenging since the user had to remember the direction of two wind circulations and five other major wind patterns. It was observed that the visual explorers reacted completely differently from the haptic explorers when they were given the question after the exploration of winds. Most V subjects indicated that they had not paid any attention to the directions of the winds, whereas $\mathrm{V}+\mathrm{H}$ subjects remembered some of the wind patterns quite well, especially at the locations where they were exposed to a strong force feedback.

We believe that $\mathrm{V}+\mathrm{H}$ subjects remembered the map better because their motor senses were stimulated, and they committed to memory the direction and the magnitude of the forces better. For example, it was observed that the haptic user remembered that his or her arm was being circulated in the clockwise direction, whereas it was harder for the visual user to remember which direction the circulation was. Even though the users were not told to pay attention to wind patterns, they built a haptic memory that they were later able to recall.

In experiment 2, we investigated whether haptic feedback helps the user understand the cause-and-effect relations between humidity, wind, temperature, and formation of clouds. It was observed that $\mathrm{V}+\mathrm{H}$ subjects were significantly better at identifying the locations where the temperature dropped along the path of the wind and where the wind entered into a relatively cooler region allowing the clouds to form. $\mathrm{V}$ subjects were able to approach to the same locations from any direction since they were not guided by the wind forces. For example, if 
the user follows the path A-B-C (along the direction of winds) in Fig. 10 to explore location number 3 under the guidance of forces, he/she can quickly observe that the color of the cube changes from dark to light, indicating a drop in temperature, and its size gets bigger, indicating an increase in humidity. Hence, clouds form in location number 3. On the other hand, if the force feedback is turned off and the user approaches the same location by following the path C-B-A, for example, the same conclusion cannot be derived. Other paths of exploration that do not coincide with paths of cause-and-effect relationships make it almost impossible for the user to discern any patterns.

The results of this experiment indicate that the lack of haptic guidance made it difficult for the $\mathrm{V}$ subjects to detect and retain the temperature and humidity patterns along the wind paths. This supports our hypothesis that haptic guidance leads users to observe changes in climate variables in a particular sequence that corresponds to the cause-andeffect relationships, and although the same information is there in the purely visual model, the unguided exploration makes it difficult to notice and/or retain these relationships. While V2 subjects observed the relationships along the same paths, they did not appear to learn and retain these relationships as well as the $\mathrm{V}+\mathrm{H}$ subjects.

The results of experiment 3 were consistent with the results of experiments 1 and 2. It was observed that $\mathrm{V}+\mathrm{H}$ subjects learned that clouds formed in areas of counterclockwise circulation and where the circulating wind was colliding with the terrain better than V subjects. It was observed that $\mathrm{V}+\mathrm{H}$ subjects paid attention to the bumpy texture mapped on the surface at cloud locations, whereas the $\mathrm{V}$ subjects reported making their decisions about the cloud locations based on their geographic knowledge and visual memory. The results confirm that haptics helped the user form a link between cloud formation, wind structure, and terrain even though the user was not explicitly told to pay attention to these variables.

In experiment 4 , all of the $\mathrm{V}+\mathrm{H}$ subjects noticed that the wind inside some of the rain cylinders was able to rise, whereas $\mathrm{V}$ subjects reported answering the related question randomly. In the training portion of this experiment, subjects in the $\mathrm{V}+\mathrm{H}$ group were able to recognize the relation between the wind rise and the resistive force inside the cylinders. We believe that $\mathrm{V}+\mathrm{H}$ subjects performed better at remembering the changes in temperature and humidity inside the cylinders better than $\mathrm{V}$ subjects because during exploration, the haptic feedback allowed the cursor to move in a more controlled way inside the rain tunnels. Results of this experiment show that the haptic users were significantly better than both V1 and V2 subjects in remembering the cause-and-effect relationships in rain formation even though they were not told to pay attention on it. These results confirm that the benefits of a haptic representation were not purely due to controlled exploration but that haptics aids learning and retention in other ways as well.

The second "testing" part of experiment 4 asked the users to consider a new map and to identify in which of the cylinders rain was likely to form by applying what they had learned in the training part. It was observed that the $\mathrm{V}+\mathrm{H}$ group was significantly more successful in locating the cylinders in which rain formed and matching the new cylinders with the ones in the first part. This lends further support to our hypothesis that when certain differences are rendered in haptics, they are detected and remembered better.

In experiment 5, we investigated if haptics improves the understanding and interpretation of the differences between two maps overloaded with climate information. Given the task of interpreting the changes in climate data when topography was manipulated, we observed that the $\mathrm{V}+\mathrm{H}$ subjects performed significantly better. The difference in performance was especially pronounced in the Antalya case (Section 4.5), where rain increases when the mountains are made higher as a result of counterclockwise circulating wind colliding with the manipulated terrain.

The experimental results strongly support hypotheses 1-4 in the Introduction and indicate that carefully designed haptic guidance and haptic rendering of certain data dimensions can be very instrumental in conveying causeand-effect relationships in a high-dimensional data space. The correct response average of the subjects to all questions is reported below in percentage (raw scores are used in the calculations when there is more than one type of score). The standard deviations show the variations among the questions. The difference between the groups is highly significant. It appears that observing the guided movements of a haptic user (V2) leads to better results than the visual exploration with no haptic feedback (V1). However, $(\mathrm{V}+\mathrm{H})$ is significantly better than V2, indicating that the arm movements guided by the haptic device in physical space has additional benefits in human perception and learning.

\begin{tabular}{|c|c|c|c|}
\hline & $\mathrm{V} 1-\mathrm{V} 2$ & $\mathrm{~V} 1-(\mathrm{V}+\mathrm{H})$ & $\mathrm{V} 2-(\mathrm{V}+\mathrm{H})$ \\
\hline Paired & $(56 \pm 17 \%)$ & $(56 \pm 17 \%)$ & $(71 \pm 11 \%)$ \\
t-tests & $(71 \pm 11 \%)$ & $(81 \pm 16 \%)$ & $(81 \pm 16 \%)$ \\
& $\mathrm{p}<0.05$ & $\mathrm{p}<0.005$ & $\mathrm{p}<0.05$ \\
\hline
\end{tabular}

It could be argued that once these cause-and-effect relationships and their manifestations in a certain map are known, it is possible to find better visual representations for them, such as animating the winds and the clouds. The experiments carried out by Group 2 were conducted to provide an approximate answer to this question and demonstrated that even when exactly the same exploration was carried out, with haptics, learning and retention improved. It is noteworthy that our haptics-augmented system allows the user to naturally notice the manifestations of these relationships on a map and internalize them while allowing him or her to direct the exploration. Our system accomplishes this without needing to preprocess, interpret, and detect patterns in the data first.

\section{CONCLUSION}

We designed, built, and experimented with a prototype that combines haptics and information visualization in order to improve the discovery, learning, and retention of causeand-effect relationships in a multidimensional data space. 
Our experiments confirmed that the inclusion of haptics significantly improves results. The existing system may be further improved by integrating a thermal display to render temperature changes or an auditory display for rendering gusty winds or by displaying other haptic effects such as vibration to simulate the start of rain.

\section{ACKNOWLEDGMENTS}

An earlier version of this work was presented orally at the 16th IEEE Symposium on Haptic Interfaces for Virtual Environment and Teleoperator Systems on 13-14 March 2008, in Reno, Nevada.

\section{REFERENCES}

[1] P. Tsai and B.E. Doty, "A Prototype Java Interface for the Grid Analysis and Display System (GrADS)," Proc. 14th Int'l Conf. Interactive Information and Processing Systems for Meteorology, Oceanography, and Hydrology, pp. 11-16, http://www.iges.org/ grads/grads.html, 1998.

[2] NCAR (National Center for Atmospheric Research), http://www. ncar.ucar.edu/tools/, 2008.

[3] R.B. Telfeyan, D.M. Rozema, and I. Gotchel, "Interactive Forecast Visualization Tools for the US Armed Forces," Proc. 22nd Int'l Conf. Interactive Information Processing Systems for Meteorology, Oceanography, and Hydrology, http://ams.confex.com/ams/ pdfpapers/100093.pdf, 2006.

[4] CLIMVIS (The Climate Visualization System), http://gcmd.nasa. gov/records/CLIMVIS.html, 2008.

[5] C.G. Healey, "Formulizing Artistic Techniques and Scientific Visualization for Painted Renditions of Complex Information Spaces," Proc. 17th Int'l Joint Conf. Artificial Intelligence (IJCAI '01), pp. 371-376, 2001.

[6] C. Harding, I.A. Kakadiaris, J.F. Casey, and R.B. Loftin, "Visualization of Very Large Datasets: A Multi-Sensory System for the Investigation of Geoscientific Data," Computers and Graphics, vol. 26, no. 2, pp. 259-269, 2002.

[7] W. Jeong and M. Gluck, "Multimodal Geographic Information Systems: Adding Haptic and Auditory Display," J. Am. Soc. for Information, Science and Technology, vol. 54, pp. 229-242, 2003.

[8] J. Rekimoto and M. Green, "The Information Cube: Using Transparency in 3D Information Visualization," Proc. Third Ann. Workshop Information Technologies and Systems (WITS '93), pp. 125-132, 1993.

[9] Regional Climate Model (RegCM3), http://users.ictp.it/RegCNET/ model.html, 2008.

[10] C. Ho, C. Basdogan, and M.A. Srinivasan, "Efficient Point-Based Rendering Techniques for Haptic Display of Virtual Objects," Presence, vol. 8, pp. 477-491, 1999.

[11] K. Joe, S. Hayashida, N. Nide, H. Kamo, K. Yamauchi, and E. Touma, "Gateau: A 3D Visualization System for Intuitive Analysis of Atmospheric Science," Proc. World Congress in Computer Science, Computer Eng. and Applied Computing (WORLDCOMP '06), pp. 860-866, 2006.

[12] D.A. Lawrence, L.Y. Pao, C.D. Lee, and R.Y. Novoselov, "Synergistic Visual/Haptic Rendering Modes for Scientific Visualization," IEEE Computer Graphics and Applications, vol. 24, no. 6, pp. 22-30, Nov./Dec. 2004.

[13] D.N. Gordin and R.D. Pea, "Prospects for Scientific Visualization as an Educational Tool," J. Learning Sciences, vol. 4, no. 3, pp. 249279, 1995.

[14] D.C. Edelson and D. Gordin, "Visualization for Learners: A Framework for Adapting Scientists' Tools," Computers and Geosciences, vol. 24, no. 7, pp. 507-616, 1998.

[15] R.L. Williams, M. Chen, and J.M. Seaton, "Haptics-Augmented Simple-Machine Educational Tools," J. Science Education and Technology, vol. 12, Mar. 2003.

[16] G. Sankaranarayanan, S. Weghorst, M. Sanner, A. Gillet and A. Olson, "Role of Haptics in Teaching Structural Molecular Biology," Proc. 11th IEEE Symp. Haptic Interfaces for Virtual Environment and Teleoperator Systems (HAPTICS '03), pp. 363-366, 2003.
[17] D. Feygin, M. Keehner, and F. Tendick, "Haptic Guidance: Experimental Evaluation of a Haptic Training Method for a Perceptual Motor Skill," Proc. 10th IEEE Symp. Haptic Interfaces for Virtual Environment and Teleoperator Systems (HAPTICS '02), pp. 40-47, 2002.

[18] M. Omata, M. Ishihara, M.G. Kwok, and A. Imamiya, "Haptizing Wind on a Weather Map with Reactive Force and Vibration," Proc. 10th IFIP TC13 Int'l Conf. Human-Computer Interaction (INTERACT '05), pp. 18-29, 2005.

[19] C. Lee, B.D. Adelstein, and S. Choi, "Haptic Weather," Proc. 16th IEEE Symp. Haptic Interfaces for Virtual Environment and Teleoperator Systems (HAPTICS '08), pp. 473-474, 2008.

[20] N. Yannier, "Using Haptics to Convey Cause and Effect Relations in Climate Visualization," MS thesis, Computational Sciences and Eng., Koc Univ., Sept. 2007.

[21] S.J. Lederman and R.L. Klatzky, "Hand Movements: A Window into Haptic Object Recognition," Cognitive Psychology, vol. 19, pp. $342-368,1987$

[22] S.J. Lederman and R.L. Klatzky, "Action for Perception: Manual Exploratory Movements for Haptically Processing Objects and Their Features," Hand and Brain: The Neurophysiology and Psychology of Hand Movements, p. 431, Academic Press, 1996.

[23] K. Kahol and S. Panchanathan, "Neuro-Cognitively Inspired Haptic User Interfaces," Multimedia Tools and Applications, vol. 37, pp. 15-38, 2008.

[24] C. Basdogan and M.A. Srinivasan, "Haptic Rendering in Virtual Environments," Handbook of Virtual Environments, K. Stanney, ed., pp. 117-134, Lawrence Earlbaum, 2002.

[25] D. Morris, H. Tan, F. Barbagli, T. Chang, and K. Salisbury, "Haptic Feedback Enhances Force Skill Learning," Proc. Second World Haptics Conf. (WHC '07), pp. 21-26, 2007.

[26] M.O. Ernst and M.S. Banks, "Humans Integrate Visual and Haptic Information in a Statistically Optimal Fashion," Nature, pp. 429-433, 2002.

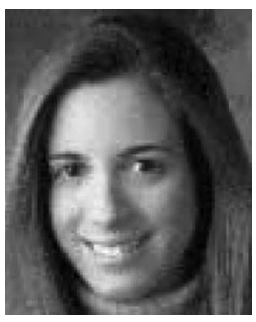

Nesra Yannier received the BS degrees in physics and computer engineering and MS degree in computational sciences and engineering from Koc University, Istanbul. She is currently a graduate student in the learning, design, and technology program at Stanford University. Her research interests include virtual environments, physically-based simulation, haptic displays and rendering, human-computer interaction, and educational technology.

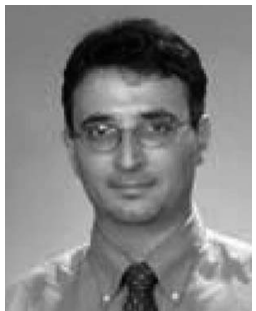

Cagatay Basdogan received the $\mathrm{PhD}$ degree in mechanical engineering from Southern Methodist University. $\mathrm{He}$ is a faculty member in the mechanical engineering and computational sciences and engineering programs in the College of Engineering, Koc University, Istanbul. Before joining Koc University, he worked at NASA-JPL/Caltech, the Massachusetts Institute of Technology, and Northwestern University Research Park. His research interests include haptic interfaces, robotics, mechatronics, biomechanics, medical simulation, computer graphics, and multimodal virtual environments. 


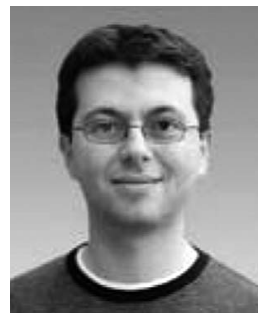

Serdar Tasiran received the PhD degree in electrical and computer engineering from the University of California, Berkeley. $\mathrm{He}$ is a faculty member in the Computer Engineering Department, College of Engineering, Koc University, Istanbul. His research interests include software engineering, software verification, concurrent systems, design automation tools for hardware and software systems, and information visualization.

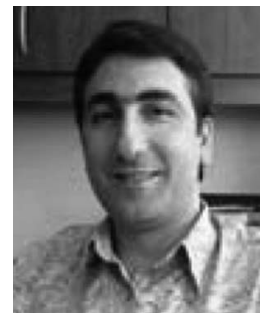

Omer Lutfi Sen received the PhD degree in hydrology from the University of Arizona. He is a faculty member in the Eurasia Institute of Earth Sciences, Istanbul Technical University, Istanbul. His research interests include land surface-atmospheric interactions, hydrometeorological modeling, regional climate modeling and anthropogenic climate change.

$\triangleright$ For more information on this or any other computing topic, please visit our Digital Library at www.computer.org/publications/dlib. 